\title{
A Flexible and Modular Framework for Implementing Infrastructures for Global Computing ${ }^{\star}$
}

\author{
Lorenzo Bettini $^{1}$, Rocco De Nicola ${ }^{1}$, Daniele Falassi ${ }^{1}$, \\ Marc Lacoste ${ }^{2}$, and Michele Loreti ${ }^{1}$ \\ 1 Dipartimento di Sistemi e Informatica, Università di Firenze \\ \{bettini, denicola, falassi,loreti\}@dsi.unifi.it \\ 2 Distributed Systems Architecture Department, France Telecom R\& D \\ marc.lacoste@rd.francetelecom.com
}

\begin{abstract}
We present a Java software framework for building infrastructures to support the development of applications for systems where mobility and network awareness are key issues. The framework is particularly useful to develop run-time support for languages oriented towards global computing. It enables platform designers to customize communication protocols and network architectures and guarantees transparency of name management and code mobility in distributed environments. The key features are illustrated by means of a couple of simple case studies.
\end{abstract}

\section{Introduction}

Technological advances of both computers and telecommunication networks, and development of more efficient communication protocols are leading to an ever increasing integration of computing systems and to diffusion of "Global Computers" [9]. These are massive networked and dynamically reconfigurable infrastructures interconnecting heterogeneous, autonomous and mobile components, that can operate on the basis of incomplete information.

Global Computers are thus fostering a new style of distributed programming that has to take into account variable guarantees for communication, cooperation, resource usage, security policies and mechanisms, and in particular code mobility [10,24]. They have stimulated the proposal of new theories, computational paradigms, linguistic mechanisms and implementation techniques for the design, realization, deployment and management of global computational environments and applications.

We have thus witnessed the birth of many calculi and kernel languages (see, e.g., [7] and the references therein) intended to support global computing programming and to provide tools for formal reasoning over the modelled systems. Many implementations of these formalisms have been proposed, and very often the language used for implementation is Java because it provides many useful features for building network applications with mobile code. However, these Java mechanisms still require a big programming effort, and so they can be thought of as "low-level" mechanisms. Because

* This work was funded by EU Global Computing initiative, project MIKADO IST-2001-32222. 
of this, many existing Java-based distributed systems (see, e.g., [1, 4, 8, 19, 22, 23] and the references therein) tend to re-implement from scratch many components that are typical and recurrent in distributed and mobile applications.

To support the implementation of languages for global computing, we have been working on a generic framework called IMC (Implementing Mobile Calculi) that can be used as a kind of middleware for the implementation of different distributed mobile systems. Such a framework aims at providing the necessary tools for implementing new language run-time systems directly derived from calculi for mobility. The basic idea and motivation of this framework is that the implementer of a new language would need concentrating on the parts that are really specific of his system, while relying on the framework for the recurrent standard mechanisms. The development of prototype implementations should then be quicker and the programmers should be relieved from dealing with low-level details. The proposed framework aims at providing all the required functionalities and abstractions for arbitrary components to communicate and move in a distributed setting.

IMC provides concrete implementations for the standard and most used functionalities that should fit most Java mobile framework requirements (e.g., Java bytecode mobility and standard network communication mechanisms). A user of IMC can customize parts of the framework by providing its own implementations for the interfaces used in the package. In this respect, the IMC framework can be straightforwardly used if no specific advanced feature is needed. The framework is however open to customizations if these are required by specific mobility systems. Customization of the framework can be achieved seamlessly by taking advantage of design patterns such as factory method, abstract factory, template method and strategy [14] that are used throughout the packages.

The framework was designed to achieve both transparency and adaptability. For instance, for code mobility, the framework provides all the basic functionalities for making code mobility transparent to the programmer: all issues related to code marshalling and code dispatch are handled automatically by the classes of the framework. Its components are designed to deal with object marshalling, code migration, and dynamic loading of code. The framework can also be adapted to deal with many network topologies (flat, hierarchical, peer-to-peer networks, etc.) and with message dispatching and forwarding. Furthermore, the implementer can build his own communication protocols by specializing the protocol base classes provided by the framework. Thus, the developer will only have to implement the parts that are relevant to the system he wants to build: typically, he will develop the communication protocol which best matches application-specific requirements. Connections and network topology are dealt with directly from within the framework. However, the developer may access the current state of his application with listeners to events that the classes of the framework generate.

The main intent of the IMC framework is not to be "yet another" distributed mobile system. It should rather be seen at a meta-level, as a framework/toolbox for building "yet another" distributed mobile system. A primordial version of the IMC framework was initiated within the MIKADO project [6]. The version we present here 
is completely re-designed and re-implemented to improve usability and to provide many additional features.

\section{Overview of the IMC Framework}

We now sketch the main functionalities and interfaces of the framework. For the sake of simplicity, we will not detail all the method signatures, e.g., we will not show the exceptions.

\subsection{Communication Protocols}

When implementing a distributed system, one of the system-specific issues is the choice of the communication protocol, which may range from high-level protocols such as Java RMI, well integrated with the Java Virtual Machine environment and taking advantage of the architectural independence provided by Java, to protocols closer to hardware resources such as TCP/IP. Marshalling strategies may range from dedicated byte-code structures to Java serialization. A generic communication framework [12, 13, $15,18,21]$ should be minimal, and allow to introduce support for new protocols with little effort, without need to re-implement a new communications library. Thus, IMC provides tools to define customized protocol stacks, which are viewed as a flexible composition of micro-protocols. The IMC design, inspired from the $x$-kernel [17] communication framework, allows to define bindings with various semantics, and to combine them in flexible ways. Thus, IMC enables to achieve adaptable forms of communication transparency, which are needed when implementing an infrastructure for global computing.

In IMC, a network protocol like, e.g., TCP, UDP, or GIOP is viewed as an aggregation of protocol states: a high-level communication protocol can indeed be described as a state automaton. The programmer implements a protocol state by extending the ProtocolState abstract class and by providing the implementation for the method enter, which returns the identifier of the next state to execute. The Protocol class aggregates the protocol states and provides the template method [14] start that will execute each state at a time, starting from the first protocol state up to the final one. Thus, the programmer must simply provide the implementation of each state, put them in the correct order in a protocol instance, and then start the protocol.

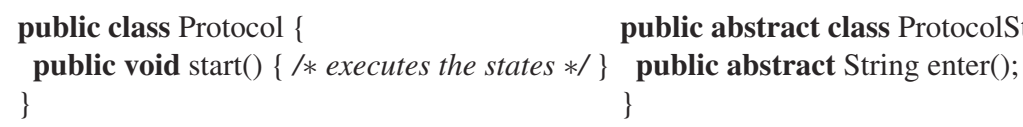

The protocol states abstract away specific communication layers. This enables reusing of a protocol implementation independently from the underlying communication means: the same protocol can then be executed on a TCP socket, on UDP packets or even on streams attached to a file (e.g., to simulate a protocol execution). This abstraction is implemented by specialized streams: Marshaller (to write) and UnMarshaller (to read). These streams provide high-level and encoding-independent representations of messages that are about to be sent or received, i.e., they are basically an extension of standard DataOutput and DataInput Java streams, with the addition of means to send 
and receive migrating code (explained later) and serialize and deserialize objects. The interface of UnMarshaller is the following (the interface of Marshaller contains the corresponding write instead of read methods):

public interface UnMarshaller extends DataInput, Closeable, MigratingCodeHandler \{

public Object readReference();

public MigratingCode readMigratingCode();

public MigratingPacket readMigratingPacket(); \}

The data in these streams can be "pre-processed" by some customized protocol layers that remove some information from the input and add some information to the output: typically this information are protocol-specific headers removed from the input and added to the output. A protocol layer is an abstract representation of a communication channel which uses a given protocol. It lets messages be sent and received through the communication channel it stands for using that protocol. The base class ProtocolLayer deals with these functionalities, and can be specialized by the programmer to provide his own protocol layer.

These layers are then composed into a ProtocolStack object that ensures the order of preprocessing passing through all the layers in the stack. For instance, the programmer can add a layer that removes a sequence number from an incoming packet and adds the incremented sequence number into an outgoing packet. The framework also provides functionalities to easily implement tunnels, so that it can be possible, e.g., to implement a tunneling layer to tunnel an existing protocol into HTTP (see Section 3).

Before reading something from a stack, a protocol state must obtain an UnMarshaller instance from the stack by calling the method up: this allows the stack layers to retrieve their own headers. In the same way, before starting to write information to the network, the state must obtain a Marshaller instance from the stack by calling the method prepare, so that the stack layers can add their own headers into the output. When the state has finished to write, it must notify the stack by calling the method down, passing the marshaller instance it had used to write the information, in order to flush the output buffer.

The methods up, prepare and down rely on methods doUp, doPrepare and doDown, respectively, of the class Protocollayer, that will be called in the right order so to implement the stack of layers. The subclasses of Protocollayer can provide their own implementations for these methods.

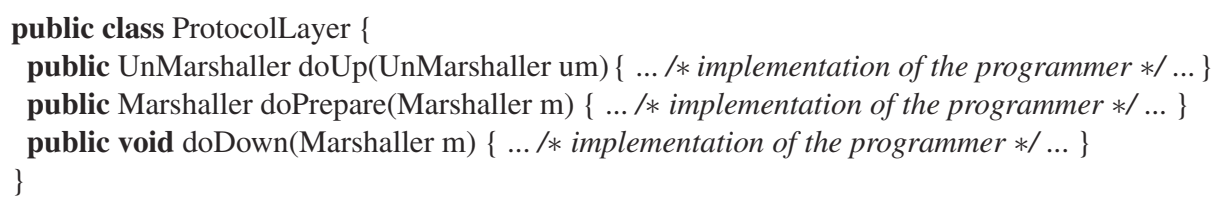

The UnMarshaller returned by the lower layer in the stack is passed to the implementation method doUp; thus, a layer can use the passed UnMarshaller to retrieve its own header and pass the UnMarshaller to the next layer, or it can create a new UnMarshaller to pass to the next layer. The latter scenario is typical of tunneling layers (as briefly shown in Section 3). Similarly, the Marshaller returned by the lower layer is passed to doPrepare. Typically, the first UnMarshaller and Marshaller 
objects will be created by the lowest layer, e.g., in case of a TCP socket, it will be a stream attached to the socket itself, while, in case of UDP packets, it will be a buffered stream attached to the datagram contents. Low layers for TCP and UDP are already provided by the framework.

\subsection{Code Mobility}

This part of the framework provides the basic functionalities for making code mobility transparent to the programmer. It deals with object marshalling, code migration, and dynamic loading of code. An object will be sent along with the byte-code of its class, and with the byte-code of all the classes of the objects it uses (i.e., all the byte-code it needs for execution). Obviously, only the code of user-defined classes must be sent, as other code (e.g., Java class libraries and the classes of the IMC packages) must be common to every application. This guarantees that classes belonging to Java standard class libraries are not loaded from other sources (especially, the network); this would be very dangerous, since, in general, such classes have many more access privileges with respect to other classes. The framework also allows the programmer to manually exclude other classes (or entire packages) from mobility.

The framework defines the empty interface MigratingCode that must be implemented by the classes representing a code that has to be exchanged among distributed sites. This code is intended to be transmitted in a MigratingPacket, stored in the shape of a byte array. How a MigratingCode object is stored in and retrieved from a MigratingPacket is taken care of by the following two interfaces:

public interface MigratingCodeMarshaller \{ public MigratingPacket marshal(MigratingCode code); \}

public interface MigratingCodeUnMarshaller \{ public MigratingCode unmarshal(MigratingPacket p); \}

Starting from these interfaces, the framework provides concrete classes that automatically deal with migration of Java objects together with their byte-code, and transparently deserialize such objects by dynamically loading their transmitted bytecode. In particular, the framework provides the base class JavaMigratingCode, implementing the above mentioned interface, MigratingCode, that provides all the procedures for collecting the Java classes that the migrating object has to bring to the remote site:

public class JavaMigratingCode extends Thread implements MigratingCode \{ public JavaMigratingPacket make_packet() $\{\ldots\}$ \}

The method make_packet will be used directly by the other classes of the framework or, possibly, directly by the programmer, to build a packet containing the serialized (marshalled) version of the object that has to migrate together with all its needed bytecode. Thus, this method will actually take care of all the code collection operations. The names of user defined classes can be retrieved by means of class introspection 
(Java Reflection API). Just before dispatching a process to a remote site, a recursive procedure is called for collecting all classes that are used by the process when declaring: data members, objects returned by or passed to a method/constructor, exceptions thrown by methods, inner classes, the interfaces implemented by its class, the base class of its class. Once these class names are collected, their byte code is gathered and packed along with the object in a JavaMigratingPacket object (a subclass of MigratingPacket storing the byte-code of all the classes used by the migrating object, besides the serialized object itself).

Finally, two classes, implementing the above mentioned interfaces MigratingCodeMansilamigratingCodeUnMarshaller, will take care of actually marshalling and unmarshalling a JavaMigratingPacket containing a migrating object and its code. In particular, the former will basically rely on the method make_packet of JavaMigratingCode, while the latter will rely on a customized class loader provided by the framework (a NodeClassLoader) to load the classes stored in the JavaMigratingPacket and then on Java serialization to actually deserialize the migrating code contained in the packet.

The readMigratingCode method of the UnMarshaller, shown in Section 2.1, will rely on an a MigratingCodeUnMarshaller to retrieve a migrating object and the corresponding method in Marshaller will rely on a MigratingCodeMarshaller to send a migrating object, so that all the code mobility issues will be dealt with internally by the framework. Even in this case, the programmer can provide his own implementations of MigratingCodeUnMarshaller and MigratingCodeMarshaller so that the framework will transparently adapt to the customized code mobility. For further details and examples concerning this part of the framework we refer the reader to [2].

\subsection{Node Topology}

The framework already provides some implemented protocols to deal with sessions. The concept of session is logical, since it can then rely on a physical connection (e.g., TCP sockets) or on a connectionless communication layer (e.g., UDP packets). In the latter case, a keep-alive mechanism can be implemented. A SessionManager instance will keep track of all the connections.

This can be used to implement several network topology structures: a flat network where only one server manages connections and all the clients are at the same level; a hierarchical network where a client can be in turn a server and where the structure of the network can be a tree or, in general, an acyclic graph of nodes; or, a peer-to-peer network.

A participant to a network is an instance of the class Node contained in the framework. A node is also a container of running processes that can be thought of as the computational units. The framework provides all the means for a process to access the resources contained in a node and to migrate to other nodes. Thus, a developer of a distributed and mobile code system has all the means to start to implement its own infrastructure or the run-time system for a mobile code language.

A process is a subclass of the class NodeProcess that implements the JavaMigratingCode base class (this allows to easily migrate a process to a 
remote site), and can be added to a node for execution with the method addProcess of the class Node.

A NodeProcess has the following interface:

public abstract class NodeProcess extends JavaMigratingCode \{

public abstract void execute();

public final void run() \{ // framework initialization operations; then call execute() \} \}

Thus, a node keeps track of all the processes that are currently in execution. A concurrent process is started by calling start on the NodeProcess thread; the final implementation of run will initialize the process structure (not detailed here) and then invoke execute that must be provided by the programmer.

A different kind of process, called node coordinator, is allowed to execute privileged actions, such as establishing a session, accepting connections from other nodes, closing a session, etc. Standard processes are not given these privileges, and this allows to separate processes that deal with node configurations from standard programs executing on nodes. For these processes a specialized class is provided called NodeCoordinator.

The programmer can provide its implementation of the concept of NodeLocation to address in a unique way a node in the net (e.g., the standard IP address:port representation). If there is a session with a node, then a location is mapped by the session manager into a protocol stack. Thus a process can retrieve a stack to run its own protocols with a remote node.

The framework also provides means to "manipulate" a protocol: it permits extending a protocol automaton by adding new states and extending the protocol stack by inserting new layers. With respect to the manipulation of the protocol automaton, it is possible to add a new starting state and a new final state, so that the original protocol is embedded in an extended protocol. When a new start and a new end state are added to an existing protocol, the framework will also take care of re-naming the previous start and end

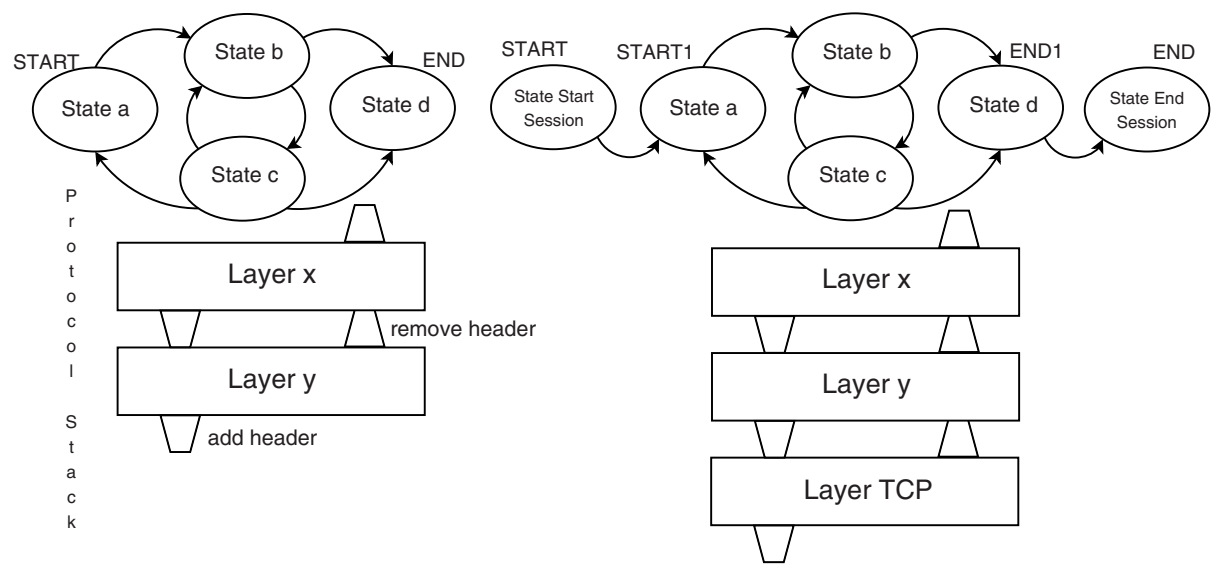

Fig. 1. The original protocol (left) and the new protocol extended with a new start and end state and the TCP layer (right) 
state and update all the references to the original start and end state with the re-named version. This will guarantee that the original protocol will transparently work as before internally, while from the outside, the new start state will be executed before the original start state and the new end state will be executed after the original end state.

The manipulation of a protocol is used internally by the classes of the framework, for instance in session management. The Node class provides a connect method to establish a session and a method accept to accept a session. These methods, apart from the connection details (e.g., host and port) also take a protocol instance as a parameter. These methods will take care of establishing (accepting, resp.) a physical connection, add a starting session protocol state as the new start state and a ending session state as the end state to the passed protocol. They also take care of setting the low layer in the protocol stack (e.g., TCP socket or UDP datagrams). Then, the protocol can be started. This manipulation is depicted in Figure 1.

\subsection{Naming and Binding}

The framework also supports logical name management, inspired by the JONATHAN ORB [12], which provides very flexible primitives to implement the concepts defined in the CORBA naming service [20]. This part of the framework aims to define a uniform manner to designate and interconnect the set of objects involved in the communication paths between computational nodes.

In IMC, an identifier is a generic notion of name that uniquely designates an object in a given naming context. Identifier semantics are naming context-specific: distributed, persistent, etc. A naming context provides name creation and management facilities. It guarantees that each of the names it controls designates some object unambiguously. It generally maps a name to an object or entity designated by that name, or can also map names to other contexts, if the resolution of names needs to be refined. Finally, a binder is a a special kind of naming context that, for a given managed name, is able to create an access path, also called binding, towards the object designated by that name.

These definitions offer a generic and uniform view of bindings, and clearly separate object identification from object access: in a given naming context $\mathrm{nc}$, a new name for an object $o$ is obtained by the nc.export (o) invocation. Chains of identifiers can then be created by exporting that name to other naming contexts. Moreover, the creation of an access path to object o designated by identifier id is performed by the id. bind () invocation which returns a ready-to-use surrogate to communicate with o. These abstractions are reflected in the following Java interfaces:

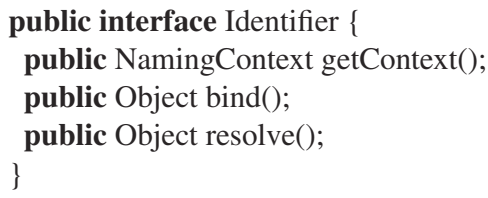

public interface NamingContext \{ public Identifier export(Object obj);

The Identifier interface represents the generic notion of identifier described above. It contains a reference to its naming context, and bears the fundamental bind operation to set up a binding between two (possibly remote) objects. The interface, using the resolve method, also permits returning the next element in a chain of identifiers, where 
each identifier was obtained as the result of exporting the next one to some naming context.

An object implementing the NamingContext interface stands for the most generic notion of a naming context which manages names of type Identifier. The interface includes the export operation to create a new name in a given context - which can, if used repeatedly, create chains of identifiers of arbitrary length. Other methods, not represented here, deal with identifier transmission over the network, using encodingindependent representations, namely involving the Marshaller and UnMarshaller interfaces already described.

This export-bind pattern is closely related to the communication part of IMC: a Protocol object can be viewed as a binder which exports (i.e., builds an access path to) a communication end-point, a Protocollayer designated through a specific type of identifier, namely a protocol layer identifier. Typically, the export operation will be called by a server object to advertise its presence on the network. This will be translated into a call to the accept method of a Node object, to accept incoming network connections. The bind operation will be called by a client-side object to bind to the interface designated by a given identifier. This will be translated into a call to the connect method of the Node object, to establish the communication path to the remote server-side object.

\section{Some Examples}

In this section we will present some simple examples that show how the framework can be used to program a customized protocol. We will not show all the details of the code, but we concentrate on how the single objects developed by the programmer can be composed together and used from within the framework itself.

First of all, in Listing 1 we show a protocol layer that removes a sequence number from the input stream and writes the incremented sequence number in the output stream. Thus, when a protocol state starts reading, this layer will remove this header and when a state starts writing this layer will add the incremented sequence number. We can create our protocol stack with this layer:

ProtocolStack mystack $=$ new ProtocolStack () ; mystack.insertLayer(new IncrementProtocolLayer());

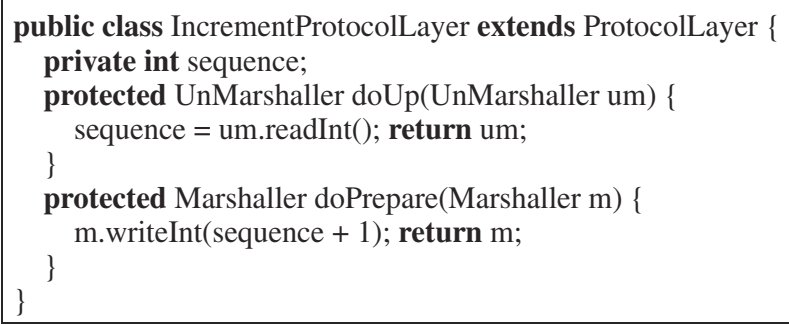

Listing 1: A protocol layer that deals with sequence numbers 


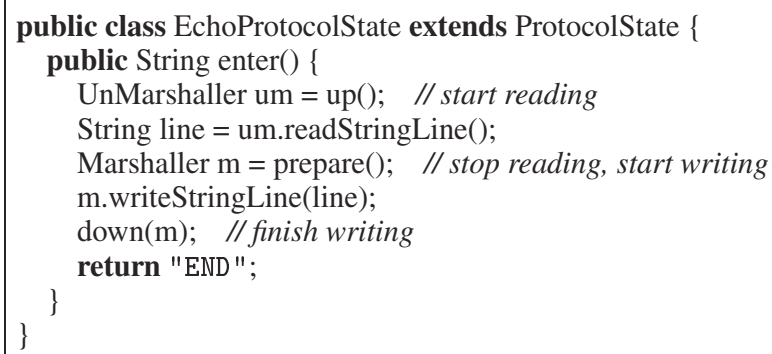

\section{Listing 2: An echo protocol state}

We can now implement our own protocol; for simplicity it will consist of only one state, that does nothing but read a line and send it back (an echo server); after that the protocol ends. In order to implement such a state, we only need to extend the ProtocolState base class and provide the implementation for the method enter and return the state END as the next state in the protocol (Listing 2). We can then create our protocol instance, set the protocol stack, and add the start state:

Protocol myprotocol = new Protocol();

myprotocol.setStack(mystack);

myprotocol.setState("START", new EchoProtocolState());

The protocol is now built, but no communication layer has been set yet. In order to do so, we can use the Node class functionalities:

Node mynode $=$ new $\operatorname{Node}()$;

mynode.accept $(9999$, myprotocol $)$;

myprotocol.start();

These instructions wait for an incoming connection on port 9999, update the protocol with a starting connection state and a final disconnection state, and update the protocol stack with the low communication layer. At this point, the protocol can start on the established physical connection.

As we suggested in Section 2, the framework provides a specialized protocol layer base class, TunnelProtocollayer, that permits implementing a tunneling layer, for enveloping a protocol inside another one. A typical example is that of an http tunnel that wraps a protocol in HTTP requests and responses. Notice that a tunnel layer does not simply remove a header when reading and add a header when writing: typically it will need to read an entire message, strip the tunneling protocol information, and pass the wrapped information to the upper layer; in the same way, it will need to intercept the information written by the upper layer and wrap it into a message according to the tunneling protocol. For this reason the framework provides this specialized base class with the features to implement these more complex functionalities. In particular, TunnelProtocollayer provides two piped stream pairs to allow the tunnel layer to communicate with the tunneled layer: the field tunneledMarshaller is piped with the field newUnMarshaller (i.e., anything written into tunneledMarshaller can be read from newUnMarshaller). Thus, the tunnel layer can implement the doUp as follows: 


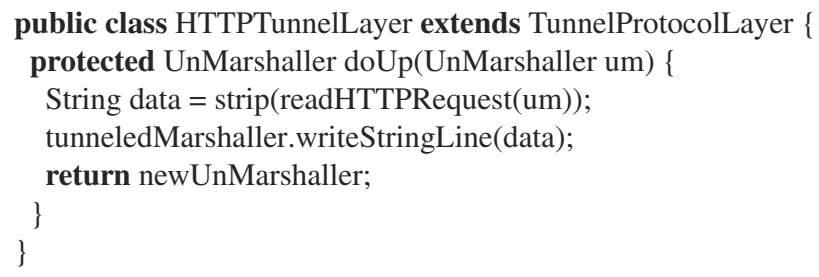

Similarly the implementation of doPrepare will return to the tunneled layer a piped UnMarshaller and doDown will read the data written by the tunneled layer from the other end of the pipe, envelop the data in the tunnel protocol structure and pass everything to the lower layer by using the Marshaller originally returned by the lower layer's prepare method.

Since a tunneling layer is still a layer, it can be inserted smoothly in an existing protocol stack:

ProtocolStack mystack = new ProtocolStack () ; mystack.insertLayer(new IncrementProtocolLayer()); mystack.insertLayer(new HTTPTunnelLayer());

Let us stress that the insertion of the tunnel layer did not require any change to the existing protocol states and layers.

\section{Conclusions}

We have presented a Java software framework for building infrastructures to support the development of applications over global computers where mobility and network awareness are key issues. The framework enables platform designers to customize communication protocols and network architectures and is particularly useful to develop run-time supports for languages oriented towards global computing.

The components have been designed after a close analysis of proposed models for mobile computing $[3,7]$. We have tried to single out the most recurrent notions of network aware programming and packed them together, to permit developers to concentrate on the really specific of their system, while relying on the framework for the recurrent standard mechanisms (node topology, communication and mobility of code).

The main aim of the framework is speeding up the development of prototype implementations and relieving programmers from low level details. Of course, if applications require a specific functionality that is not in the framework (e.g., a customized communication protocol built on top of TCP/IP, or a more sophisticated mobile code management), programmers can customize the part relative to these mechanisms. The presented framework will be shortly released as open source software.

It has been pointed out that our design has some similarities with .Net Remoting, which provides an abstract approach to interprocess communication and separates the "remotable" object from a specific client or server application domain and from a specific mechanism of communication. A closer look at the relationships between the two approaches is definitely on demand. These relations are now under investigation.

In the near future, we will also use the framework for implementing $D \pi[16]$ and for re-engineering KLAVA [5], the run time support for KLAIM [11] and for implementing 
richer languages for global computing. But, we plan also to extend the IMC components to deal with security issues.

Acknowledgments. We are grateful to all people involved in the MIKADO project, in particular, we would like to thank L. Lopes and V. Vasconcelos that contributed to the initial design of IMC.

\section{References}

1. A. Acharya, M. Ranganathan, and J. Saltz. Sumatra: A Language for Resource-aware Mobile Programs. In Vitek and Tschudin [25], pages 111-130.

2. L. Bettini. A Java Package for Transparent Code Mobility. In Proc. FIDJI, LNCS 3409, pages 112-122. Springer, 2004.

3. L. Bettini, M. Boreale, R. De Nicola, M. Lacoste, and V. Vasconcelos. Analysis of Distribution Structures: State of the Art. MIKADO Project Deliverable D3.1.1, 2002.

4. L. Bettini, R. De Nicola, G. Ferrari, and R. Pugliese. Interactive Mobile Agents in X-KLAIM. In Proc. WETICE, pages 110-115. IEEE, 1998.

5. L. Bettini, R. De Nicola, and R. Pugliese. KLAVA: a Java Package for Distributed and Mobile Applications. Software - Practice and Experience, 32(14):1365-1394, 2002.

6. L. Bettini, D. Falassi, R. D. Nicola, M. Lacoste, L. Lopes, M. Loreti, L. Oliveira, H. Paulino, and V. Vasconcelos. Language Experiments v1: Simple Calculi as Programming Language. MIKADO Project Deliverable D3.2.1, 2004.

7. G. Boudol, I. Castellani, F. Germain, and M. Lacoste. Models of Distribution and Mobility: State of the Art. MIKADO Project Deliverable D1.1.1, 2002.

8. G. Cabri, L. Leonardi, and F. Zambonelli. Reactive Tuple Spaces for Mobile Agent Coordination. In K. Rothermel and F. Hohl, editors, Proc. 2nd Int. Workshop on Mobile Agents, LNCS 1477, pages 237-248. Springer, 1998.

9. L. Cardelli. Abstractions for Mobile Computation. In J. Vitek and C. Jensen, editors, Secure Internet Programming: Security Issues for Mobile and Distributed Objects, LNCS 1603, pages 51-94. Springer-Verlag, 1999.

10. G. Cugola, C. Ghezzi, G. Picco, and G. Vigna. Analyzing Mobile Code Languages. In Vitek and Tschudin [25].

11. R. De Nicola, G. Ferrari, and R. Pugliese. KLAIM: a Kernel Language for Agents Interaction and Mobility. IEEE Transactions on Software Engineering, 24(5):315-330, 1998.

12. B. Dumant, F. Horn, F. Dang Tran, and J.-B. Stefani. Jonathan: an Open Distributed Processing Environment in Java. In Proc. MIDDLEWARE, 1998.

13. ExoLab Group. The OpenORB project, 2002. http://openorb. exolab.org/.

14. E. Gamma, R. Helm, R. Johnson, and J. Vlissides. Design Patterns: Elements of Reusable Object-Oriented Software. Addison-Wesley, 1995.

15. R. Hayton, A. Herbert, and D. Donaldson. Flexinet: a Flexible Component Oriented Middleware System. In Proc. ACM SIGOPS European Workshop, 1998.

16. M. Hennessy and J. Riely. Resource Access Control in Systems of Mobile Agents. In U. Nestmann and B. C. Pierce, editors, HLCL '98, volume 16.3, pages 3-17. Elsevier, 1998.

17. N. Huntchinson and L. Peterson. The $x$-kernel: an Architecture for Implementing Network Protocols. IEEE Transactions on Software Engineering, 17(1):64-76, 1991.

18. R. Klefstad, D. Schmidt, and C. O'Ryan. The Design of a Real-time CORBA ORB using Real-time Java. In Proc. ISORC, 2002.

19. D. Lange and M. Oshima. Programming and Deploying Java Mobile Agents with Aglets. Addison-Wesley, 1998. 
20. Object Management Group. Naming Service Specification, version 1.3 edition, 2004. Available at http://www .omg. org.

21. C. O'Ryan, F. Kuhns, D. Schmidt, O. Othman, and J. Parsons. The Design and Performance of a Pluggable Protocols Framework for Real-time Distributed Object Computing Middleware. In Proc. MIDDLEWARE, 2000.

22. H. Peine and T. Stolpmann. The Architecture of the Ara Platform for Mobile Agents. In Proc. MA, LNCS 1219, pages 50-61. Springer, 1997.

23. G. Picco, A. Murphy, and G.-C. Roman. LiME: Linda Meets Mobility. In D. Garlan, editor, Proc. ICSE, pages 368-377. ACM Press, 1999.

24. T. Thorn. Programming Languages for Mobile Code. ACM Computing Surveys, 29(3):213239, 1997. Also Technical Report 1083, University of Rennes IRISA.

25. J. Vitek and C. Tschudin, editors. Mobile Object Systems - Towards the Programmable Internet, LNCS 1222. Springer, 1997. 